\title{
A hydrodynamic scheme for two-component winds from hot stars
}

\author{
V. Votruba ${ }^{1,3}$, A. Feldmeier ${ }^{2}$, J. Kubát ${ }^{1}$, and D. Rätzel ${ }^{2}$ \\ 1 Astronomický ústav, Akademie věd České republiky, 25165 Ondřejov, Czech Republic \\ e-mail: votruba@sunstel.asu.cas.cz \\ 2 Astrophysik, Institut für Physik, Universität Potsdam, Am Neuen Palais 10, 14469 Potsdam, Germany \\ e-mail: afeld@astro.physik.uni-potsdam.de \\ 3 Ústav teoretické fyziky a astrofyziky, Přirodověcká fakulta, Masarykova univerzita, Kotlářská 2, 61137 Brno, Czech Republic
}

Received 11 September 2006 / Accepted 21 June 2007

\section{ABSTRACT}

\begin{abstract}
Aims. We have developed a time-dependent two-component hydrodynamics code to simulate radiatively-driven stellar winds from hot stars.

Methods. We use a time-explicit van Leer scheme to solve the hydrodynamic equations of a two-component stellar wind. Dynamical friction due to Coulomb collisions between the passive bulk plasma and the line-scattering ions is treated by a time-implicit, semianalytic method using a polynomial fit to the Chandrasekhar function. This gives stable results despite the stiffness of the problem. Results. This method was applied to model stars with winds that are both poorly and well-coupled. While for the former case we reproduce the mCAK solution, for the latter case our solution leads to wind decoupling.
\end{abstract}

Key words. stars: winds, outflows - hydrodynamics - instabilities

\section{Introduction}

Stellar winds from hot stars are often described using the socalled CAK theory (Castor et al. 1975), including later improvements for a finite stellar disk (Pauldrach et al. 1986; Friend $\&$ Abbott 1986) and the ionisation stratification of the wind (Abbott 1982). The radiative line force is here parameterised by three constants only, $k, \alpha$, and $\delta$.

Although qualitative agreement between theory and observations was achieved, there were still discrepancies remaining, namely the terminal velocity $v_{\infty}$ being too low and the mass-loss rate $\dot{M}$ being too high (Abbott 1982; Friend \& Abbott 1986). Besides this, calculations did not reproduce the observed ionisation ratios (Pauldrach \& Puls 1990). Further development of the theory was focused mainly on the effect of multiline scattering, magnetic field and rapidly rotating stars. As shown by Friend \& Castor (1983), if one includes the effect of multiple scatterings in overlapping lines, the terminal velocity is higher than the CAK value, while the mass-loss rate remains similar to the latter. Furthermore, the inclusion of a magnetic field and the assumption of high rotational velocity lead to a higher terminal velocity and a higher mass-loss rate (Friend \& MacGregor 1984).

The above problem with the ionisation structure can be resolved by including X-rays. X-rays are a common feature among the $\mathrm{O}$ stars and can change the ionisation structure of the wind significantly. As was first discussed by (Cassinelli \& Olson 1979) and later by (Pauldrach \& Puls 1990), the X-rays lead to photoinisation via Auger processes and are necessary to explain the presence of superionised ions O VI in observations.

Lucy \& Solomon (1970) suggested a new hydrodynamic instability of the radiative driving force. This so-called linedriven instability can possibly explain observed variabilities of stellar winds, such as variable X-ray emission, discrete absorption components, and the appearance of broad absorption troughs in P Cygni line profiles (Prinja \& Howarth 1986). The contradictory results from the simplified linear stability analysis of MacGregor et al. (1979) and Carlberg (1980), on one hand, and Abbott (1980), on the other, were unified by Owocki \& Rybicki (1984, 1985) in the so-called bridging law. The first time-dependent, numerical simulations of the instability were undertaken by Owocki et al. (1988), who found that the wind develops a train of strong reverse shocks. Their model was improved by Feldmeier (1995), including the energy equation without the approximation of gas isothermality to calculate the temperature structure of the stellar wind. Adopting a simple formulation for the line force, Dessart \& Owocki (2005) extended hydrodynamical instability simulations from one to two dimensions (2D), to find the lateral coherence length of instabilitygenerated shells of dense gas in the wind.

The approximation of the one-component flow assumed in the CAK model is acceptable for sufficiently dense winds from $\mathrm{O}$ stars and hot B stars (Castor et al. 1976). The radiative line force acts only on ions (termed "metals" in the following) that scatter photons in numerous spectral lines. These ions share their momentum through Coulomb collisions with the passive part of the plasma (protons). The dynamical effect of Coulomb collisions on the plasma is described well by dynamical friction, which was introduced by Chandrasekhar (1943) for the gravitational force and later applied to electromagnetic forces by Spitzer (1956).

For thin winds from B near-main-sequence stars, however, decoupling and a well-known plasma runaway effect can occur (Springmann \& Pauldrach 1992). At low wind densities, Coulomb interactions are weak, and the momentum transfer from metals to protons becomes inefficient. As a result, the two components decouple at a given radius. From this decoupling radius on, metals are strongly accelerated by the radiative force, whereas the passive plasma is decelerated by gravity.

It came as a surprise that Krtička \& Kubát (2000) obtained a different result from stationary hydrodynamic calculations. They found a wind solution where the two components remain 
coupled, but undergo a sudden jump to a slow-acceleration branch at a definite radius. This is analogous to what is found for shallow (and overloaded) solutions (Feldmeier \& Shlosman 2000). The issue has not been settled yet ever since Owocki \& Puls (2002) showed from linear stability analysis that the flow should be disrupted by ion separation before reaching a slowacceleration solution. Krtička \& Kubát (2001b) also found that in a low-density wind, frictional heating may be important for the energy balance of the whole wind and may increase the temperature of the gas significantly.

An effect similar to ion decoupling was considered by Porter \& Skouza (1999), namely shock decoupling. In a low-density wind that passes through a shock, the postshock gas remains at high temperatures and in a highly ionised state because the gas is too rarefied to cool radiatively (Krolik \& Raymond 1985). In this case the ions responsible for line driving are completely stripped and are not accelerated further by the radiation field.

Another interesting idea in this context is the generation of pulsating shells (Porter \& Skouza 1999). If the wind decouples at a radius where the flow speed is still lower than the escape speed, the passive plasma falls back to the star, and the interaction with the outflow leads to pulsating shells. However, this result is based only on a one-component model, with an artificial turn-off of the radiative acceleration.

To analyse the possible occurrence of ion decoupling and pulsating shells in thin stellar winds, it is necessary to develop a suitable time-dependent, two-component hydrodynamics code. In the present paper we describe our numerical method to simulate these outflows, and give basic results on thick and thin winds.

\section{Two-component winds}

We restrict ourselves to a $1 \mathrm{D}$ spherically symmetric, isothermal, quasineutral, two-component outflow consisting of metals that scatter stellar photons in numerous spectral lines and a passive plasma. The forces acting are gravity, dynamical friction, gas pressure gradients, and, for only the line-scattering ions, the radiative line force. The continuity equations are

$$
\begin{gathered}
\frac{\partial \rho_{\mathrm{p}}}{\partial t}+\frac{1}{r^{2}} \frac{\partial\left(r^{2} \rho_{\mathrm{p}} v_{\mathrm{p}}\right)}{\partial r}=0, \\
\frac{\partial \rho_{\mathrm{i}}}{\partial t}+\frac{1}{r^{2}} \frac{\partial\left(r^{2} \rho_{\mathrm{i}} v_{\mathrm{i}}\right)}{\partial r}=0,
\end{gathered}
$$

and the Euler equations are

$$
\begin{aligned}
\frac{\partial v_{\mathrm{p}}}{\partial t}+v_{\mathrm{p}} \frac{\partial v_{\mathrm{p}}}{\partial r}+\frac{1}{\rho_{\mathrm{p}}} \frac{\partial p_{\mathrm{p}}}{\partial r} & =\frac{R_{\mathrm{pi}}}{\rho_{\mathrm{p}}}-g_{\mathrm{eff}}, \\
\frac{\partial v_{\mathrm{i}}}{\partial t}+v_{\mathrm{i}} \frac{\partial v_{\mathrm{i}}}{\partial r}+\frac{1}{\rho_{i}} \frac{\partial p_{\mathrm{i}}}{\partial r} & =g_{\mathrm{rad}}^{\mathrm{i}}-g_{\mathrm{eff}}-\frac{R_{\mathrm{pi}}}{\rho_{\mathrm{i}}} .
\end{aligned}
$$

Here, $\rho_{\mathrm{i}}, v_{\mathrm{i}}$, and $p_{\mathrm{i}}$ are the density, velocity, and pressure of the metals, and $\rho_{\mathrm{p}}, v_{\mathrm{p}}$, and $p_{\mathrm{p}}$ are the density, velocity, and pressure of the passive plasma. The effective gravitational acceleration is $g_{\mathrm{eff}}=-G M_{*}\left(1-\Gamma_{\mathrm{e}}\right) / r^{2}$, with gravitational constant $G$ and Eddington factor $\Gamma_{\mathrm{e}}$. The frictional force $R_{\mathrm{pi}}$ between metals and passive plasma is described below (see Eq. (8)). The above system is closed by the equations of state,

$$
\begin{aligned}
& p_{\mathrm{p}}=\sqrt{k T / m_{\mathrm{p}}} \rho_{\mathrm{p}}, \\
& p_{\mathrm{i}}=\sqrt{k T / m_{\mathrm{i}}} \rho_{\mathrm{i}} .
\end{aligned}
$$

The assumption of wind isothermality becomes questionable in the presence of strong frictional heating, and we aim to include the energy equation in future work.

\subsection{Radiative acceleration}

We write the radiative acceleration in the form (Krtička \& Kubát 2000, Eq. (3))

$g_{\mathrm{rad}}^{\mathrm{i}}(r)=\frac{\left(\eta \sigma_{\mathrm{e}}\right)^{1-\alpha}}{4 \pi v_{\mathrm{th}}^{\alpha}} \frac{L_{*}}{r^{2}} k\left(\frac{1}{\rho_{\mathrm{i}}} \frac{\partial v_{\mathrm{i}}}{\partial r}\right)^{\alpha} f_{\mathrm{ion}} f_{\text {fin }}$,

with CAK force multipliers $k, \alpha, \delta$. Here, $L_{*}$ is the luminosity of the star, $v_{\text {th }}$ the thermal velocity of ions, and $\sigma \approx 0.33 \mathrm{~cm}^{2} \mathrm{~g}^{-1}$ the Thomson opacity due to scattering on electrons.

The radiative acceleration due to line scattering only acts on metal ions, but the CAK force multipliers $k, \alpha, \delta$ were calculated for a one-component plasma. We account for this by a scaling factor $\eta$ to the radiative force. The value $\eta=0.0127$ calculated by Krtička \& Kubát (2000) for solar metallicities is adopted here.

The finite disk correction factor $f_{\text {fin }}$ is given by (Castor et al. 1975)

$f_{\text {fin }}(r)=\frac{(1+\sigma)^{\alpha+1}-\left(1+\sigma \mu_{*}^{2}\right)}{\sigma(\sigma+1)^{\alpha}\left(1-\mu_{*}^{2}\right)(\alpha+1)}$,

where $\mu_{*}=\sqrt{1-\left(R_{*} / r\right)^{2}}$ (with stellar radius $R_{*}$ ), and $\sigma$ is given by (Castor 1974):

$\sigma=\frac{r}{v_{\mathrm{i}}} \frac{\partial v_{\mathrm{i}}}{\partial r}-1$

Finally, $f_{\text {ion }}$ is a correction for the ionisation state of the stellar wind (Abbott 1982),

$f_{\text {ion }}(r)=\left(\frac{10^{-11}\left[\mathrm{~cm}^{3}\right] n_{\mathrm{e}}}{W(r)}\right)^{\delta}$,

where $W(r)=\frac{1}{2}\left(1-\sqrt{1-\left(R_{*} / r\right)^{2}}\right)$ is the geometrical dilution factor. Due to the assumption of quasineutrality, the number density of electrons $n_{\mathrm{e}}$ roughly matches the number density of the passive plasma. Thus, we use $n_{\mathrm{e}}=n_{\mathrm{p}}$ in $f_{\text {ion }}$. This correction factor does not have a significant influence on the wind dynamics (Abbott 1982).

\subsection{Friction terms}

The passive plasma and absorbing ions interact via Coulomb collisions, which are described by a frictional force $R_{\mathrm{pi}}$ per volume (Springmann \& Pauldrach 1992),

$R_{\mathrm{pi}}=n_{\mathrm{p}} n_{\mathrm{i}} k_{\mathrm{pi}} G\left(x_{\mathrm{pi}}\right)$,

where $n_{\mathrm{p}}$ and $n_{\mathrm{i}}$ are the number densities of the passive plasma and absorbing ions, respectively, and the frictional coefficient $k_{\mathrm{pi}}$ is given by

$k_{\mathrm{pi}}=\frac{4 \pi \ln \Lambda Z_{\mathrm{p}}^{2} Z_{\mathrm{i}}^{2} e^{4}}{k_{\mathrm{B}} T} \frac{v_{\mathrm{i}}-v_{\mathrm{p}}}{\left|v_{\mathrm{i}}-v_{\mathrm{p}}\right|}$.

Here, $Z_{\mathrm{i}} e$ and $Z_{\mathrm{p}} e$ are the ion and passive plasma charges, respectively. The Coulomb logarithm $\ln \Lambda$ is defined as

$\ln \Lambda=\ln \left[\frac{24 \pi}{\sqrt{n}}\left(\frac{k_{\mathrm{B}} T}{4 \pi e^{2}}\right)^{1.5}\right]$ 
with Boltzmann constant $k_{\mathrm{B}}$, total number density $n$, and wind temperature $T$. The Chandrasekhar function $G\left(x_{\mathrm{pi}}\right)$ (Chandrasekhar 1943; Spitzer 1956) in Eq. (8) is given in terms of the error function $\Phi\left(x_{\mathrm{pi}}\right)$ by (Spitzer 1956)

$G\left(x_{\mathrm{pi}}\right)=\frac{\Phi\left(x_{\mathrm{pi}}\right)}{2 x_{\mathrm{pi}}^{2}}-\frac{\exp \left(-x_{\mathrm{pi}}^{2}\right)}{x_{\mathrm{pi}} \sqrt{\pi}}$.

This function depends on $x_{\mathrm{pi}}$, the ion separation drift speed relative to the passive plasma, scaled to the mass-weighted thermal speed (Springmann \& Pauldrach 1992)

$x_{\mathrm{pi}}=\frac{\left|v_{\mathrm{i}}-v_{\mathrm{p}}\right|}{v_{\mathrm{th}} \sqrt{1+A_{\mathrm{i}} / A_{\mathrm{p}}}}$,

where $A_{\mathrm{i}}$ and $A_{\mathrm{p}}$ represent the mean atomic mass of ions and passive plasma in atomic units.

\section{Method of solution}

To solve the four hydrodynamic equations we use a hydrodynamics code developed by Feldmeier (1995) as a core. It employs a standard Euler scheme and is suited for $1 \mathrm{D}$, onecomponent outflows. We extended this code to a two-component version. Equations (1a) and (2) are discretised using an operatorsplitting, time-explicit, finite difference method on a staggered mesh (see LeVeque et al. 1998, p. 131). We calculate advection fluxes using van Leer's monotonic interpolation (see van Leer 1982).

Krtička \& Kubát (2000) discussed the circumstances under which the radiative line acceleration is balanced by dynamical friction and not by inertia. In this case, a decrease in dynamical friction leads (counter-intuitively) to a decrease in the radiative acceleration of the gas. We accelerate the gas by the sum of dynamical friction and radiative force, i.e. avoid operator-splitting of these two force terms, in order to achieve a stable numerical scheme. Similarly, for a barometric density stratification, one has to apply the sum of the thermal pressure force and gravity at once to avoid an unstable scheme.

As the time step, we use the minimum of the separate Courant time steps for the two flow components. The wind is characterised by two parameters, the mass-loss rate and terminal speed. The latter scales with the escape speed, and the former is given roughly by the CAK relation (Castor et al. 1975, Eq. (46))

$\dot{M}_{\mathrm{CAK}}=\frac{4 \pi G M}{\sigma_{\mathrm{e}}^{\mathrm{ref}} v_{\text {th }}} \alpha(1-\alpha)^{(1-\alpha) / \alpha}\left(k \Gamma_{\mathrm{e}}\right)^{1 / \alpha}\left(1-\Gamma_{\mathrm{e}}\right)^{-(1-\alpha) / \alpha}$.

To calculate the radiative force we use tabulated values of the CAK parameters from Abbott (1982).

As initial conditions for the case of a well-coupled wind we use, for the velocity of the both components in the subsonic part, $v(r)=0.1 a \exp (H r)$, where $H$ is the scale height and $a$ the isothermal sound speed. In the supersonic part, we use $v(r)=a+20 a r$. Initial values for the density of both components are calculated from Eqs. (1a) and (13).

For the case of a low-density wind (where poor coupling between the components may be expected), we artificially increase the frictional coupling by increasing the average ion charge $q_{\mathrm{i}}$ and run the simulation (with the above initial conditions) until a converged CAK one-component solution is achieved. This solution then serves as initial conditions for both components in a subsequent simulation with a realistic average ion charge.

Our boundary conditions are set according to the theory of characteristics (Anderson 1995, pp. 303-307). For absorbing

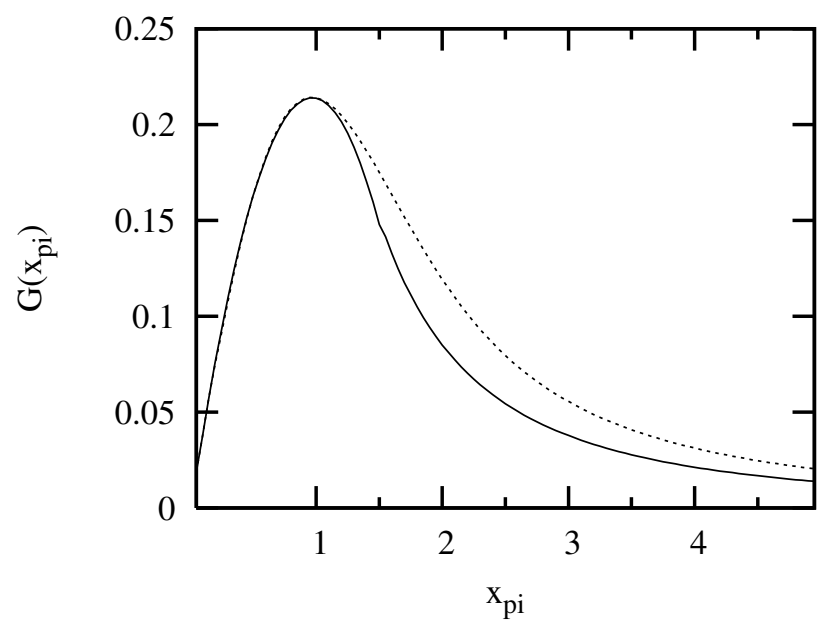

Fig. 1. The Chandrasekhar function: exact function $G\left(x_{\mathrm{pi}}\right)$ according to (11) (dashed line), and approximation $G_{A}\left(x_{\mathrm{pi}}\right)$ (solid line).

ions and passive plasma, we keep the densities $\rho_{\mathrm{p}}$ and $\rho_{\mathrm{i}}$ at the inner boundary fixed to their initial values and set, at each time step, the momentum densities $\rho_{\mathrm{i}} v_{\mathrm{i}}, \rho_{\mathrm{p}} v_{\mathrm{p}}$ at the inner boundary to their value at the first interior mesh point (zero-order extrapolation). At the outer boundary, we extrapolate the mass and momentum densities of both components from the last interior mesh point.

\subsection{Discretisation of friction}

The central issue of the present paper is the inclusion of the frictional term (8). In the following we develop a simple, computationally effective, and accurate method for determining of the velocity difference $x_{\mathrm{pi}}$ at every time step, based on the frictional term.

The equations for the momentum change of the passive plasma and the absorbing ions due to the frictional term alone are, from (2)

$$
\begin{aligned}
\frac{\partial v_{r, \mathrm{i}}}{\partial t} & =-\frac{C G\left(x_{\mathrm{pi}}\right)}{\rho_{\mathrm{p}}}, \\
\frac{\partial v_{r, \mathrm{p}}}{\partial t} & =\frac{C G\left(x_{\mathrm{pi}}\right)}{\rho_{i}}
\end{aligned}
$$

where the constant $C$ is given by

$$
C=-\frac{k_{\mathrm{pi}}}{A_{\mathrm{i}} A_{\mathrm{p}} m_{\mathrm{p}}^{2}} .
$$

Transforming the above differential equations into time-explicit difference equations results in a system with high stiffness, since we attempt to evolve a stellar wind on a relatively slow flow time scale with respect to which the faster, frictional processes maintain equilibrium almost instantaneously. As a consequence, the numerical solution fails due to large oscillations at the wind base. Therefore we set up the following semi-implicit scheme for the frictional terms. Subtracting Eq. (15) from Eq. (14), we obtain a differential equation for the drift velocity,

$$
\frac{\mathrm{d} x_{\mathrm{pi}}}{\mathrm{d} t}=-\frac{\left(\rho_{\mathrm{i}}+\rho_{\mathrm{p}}\right) C}{v_{\mathrm{th}} \sqrt{1+A_{\mathrm{i}} / A_{\mathrm{p}}}} G\left(x_{\mathrm{pi}}\right) .
$$

In Eqs. (14) and (15) we now replace the partial time derivatives by a total derivative following the idea of operator-splitting, i.e., 
friction is now treated without reference to any "forthcoming" hydrodynamic processes. The $\mathrm{d} / \mathrm{d} t$ is not to be understood as a Lagrange derivative. Upon formal integration,

$\int_{x_{\mathrm{pi}, 0}}^{x_{\mathrm{p}, 1}} \frac{\mathrm{d} x_{\mathrm{pi}}}{G\left(x_{\mathrm{pi}}\right)}=-\int_{t_{0}}^{t_{0}+\Delta t} C \frac{\left(\rho_{\mathrm{i}}+\rho_{\mathrm{p}}\right)}{v_{\mathrm{th}} \sqrt{1+A}} \mathrm{~d} t$,

where $A$ denotes the ratio $A_{\mathrm{i}} / A_{\mathrm{p}}$, and $x_{\mathrm{pi}, 0}$, resp. $x_{\mathrm{pi}, 1}$, are the drift velocities at times $t_{0}$, resp. $t_{0}+\Delta t$. We want to obtain an analytical expression for the left hand side of Eq. (18). To this end we use a three-interval fit to the Chandrasekhar function. For low and high drift velocities we use the same approximations as Owocki \& Puls (2002),

$G_{1}\left(x_{\mathrm{pi}}\right) \approx \frac{2 x_{\mathrm{pi}}}{3 \sqrt{\pi}}$ for $x_{\mathrm{pi}} \leq x_{1}$

and

$G_{3}\left(x_{\mathrm{pi}}\right) \approx \frac{K}{2 x_{\mathrm{pi}}^{2}} \quad$ for $x_{\mathrm{pi}} \geq x_{2}$.

For values between $x_{1}$ and $x_{2}$, we approximate the Chandrasekhar function using a quadratic function,

$G_{2}\left(x_{\mathrm{pi}}\right) \approx a_{2} x_{\mathrm{pi}}^{2}+b_{2} x_{\mathrm{pi}}+c_{2}$,

where the parameters $a_{2}, b_{2}, c_{2}$ are evaluated from the conditions $G_{1}\left(x_{1}\right)=G_{2}\left(x_{1}\right), G_{2}\left(x_{\max }\right)=0.214$, and $\mathrm{d} G_{1}\left(x_{1}\right) / \mathrm{d} x=$ $\mathrm{d} G_{2}\left(x_{1}\right) / \mathrm{d} x$. Continuity of the derivative at $x_{2}$ is not required, because we have only a second-order polynomial approximation. The points $x_{1}, x_{2}$, and the scaling factor $K$ were chosen to achieve the best fit to the Chandrasekhar function (11). We find, for $x_{1}=0.1$ and $x_{2}=1.5$, the values $a_{2}=-0.2341$, $b_{2}=0.4532, c_{2}=-0.0053$ and $K=0.74$. This approximation to the Chandrasekhar function is termed $G_{A}$ in the following and is shown in Fig. 1.

For a well-coupled wind the difference between $G$ and $G_{A}$ is of minor importance, and both functions lead to essentially the same wind solution. To show this, we use two different approximations $G_{A}$, one being a global overestimate, the other a global underestimate of $G$. In both cases, the code converged to almost the same steady CAK solution.

Using the approximation $G_{A}$, we obtain the velocity difference $x_{\mathrm{pi}}$ as follows. At the dynamically most important part of the Chandrasekhar function, i.e. its maximum (covered by $G_{2}$ ), integration of (18) leads to

$\left.\ln \frac{2 a_{2} x_{\mathrm{pi}}+b_{2}-\Xi}{2 a_{2} x_{\mathrm{pi}}+b_{2}+\Xi}\right|_{x_{\mathrm{p}, 0}} ^{x_{\mathrm{p}, 1}}=-C \Delta t \Xi$,

where $\Xi=\sqrt{b_{2}^{2}-4 a_{2} c_{2}}$. With the help of the substitutions

$q_{-}=2 a_{2} x_{\mathrm{pi}, 0}+b_{2}-\Xi$,

$q_{+}=2 a_{2} x_{\mathrm{pi}, 0}+b_{2}+\Xi$,

we can simplify the expression (22) and as final form of the velocity difference obtain

$x_{\mathrm{pi}, 1}=\frac{1}{2 a_{2}}\left[\frac{1+\frac{q_{-}}{q_{+}} \exp (-C \Delta t \Xi)}{1-\frac{q_{-}}{q_{+}} \exp (-C \Delta t \Xi)} \Xi-b_{2}\right]$.

For low drift velocities and using the approximation $G_{1}\left(x_{\mathrm{pi}}\right)$, integration of (18) gives

$x_{\mathrm{pi}, 1}=x_{\mathrm{pi}, 0} \exp \left(-\frac{2}{3 \sqrt{\pi}} C \Delta t\right)$, and finally, for high drift velocities and using the approximation $G_{3}\left(x_{\mathrm{pi}}\right)$,

$x_{\mathrm{pi}, 1}=\sqrt[3]{x_{\mathrm{pi}, 0}^{3}-\frac{3}{2} K C \Delta t}$.

The expressions (25)-(27) are used in our code to calculate the change in drift velocity $x_{\mathrm{pi}}$ due to friction during a hydrodynamic time step. This results in a highly improved stability behaviour compared to direct, time-explicit differencing of the friction terms and, for the first time, allows time-dependent simulations of multi-component winds.

Finally, we note that a commonly used approximation to the Chandrasekhar function (Karlický et al. 2000)

$G_{A}\left(x_{\mathrm{pi}}\right)=\frac{K x_{\mathrm{pi}}}{\left(x_{\mathrm{pi}}^{2}+v_{\mathrm{th}}^{2}\right)^{3 / 2}}$,

which covers the whole interval of drift speeds, results in integrals we were not able to carry out analytically.

\subsection{Time requirements}

Since the two-component code is essentially a duplication of the one-component code, and since the frictional force terms are solved by analytic equations, i.e. at very small computational cost, the cpu time for a simulation is comparable to the onecomponent case, typically a few hours (for $O\left(10^{3}\right)$ mesh points) on a dedicated workstation. However, we chose a very small Courant number of 0.05 (instead of typically 0.5 ) to bring the inviscid Courant time step somewhat closer to the frictional time step, in order to avoid changes too large in friction terms during subsequent time steps, which could trigger instability.

\section{Results of calculations}

In the present paper, we considered essentially the same stars as Krtička \& Kubát (2000). More specifically, we apply our method to the wind where a well-coupled solution is predicted (we refer to this case as B0) and to the wind with possible decoupling (referred to as B5). The corresponding stellar parameters are given in Table 1. Details about these parameters are discussed by Krtička \& Kubát (2000).

To determine whether our model winds are well or poorly coupled, we considered the parameter $\Gamma_{\mathrm{L}}$, which is the ratio of the radiative force per mass acting on ions, $g_{\text {rad }}^{\mathrm{i}}$, to the gravitational acceleration $g$ Springmann \& Pauldrach (1992),

$\Gamma_{\mathrm{L}}=\frac{\rho_{\mathrm{i}}}{\rho_{\mathrm{i}}+\rho_{\mathrm{p}}} \frac{g_{\mathrm{rad}}^{\mathrm{i}}(r)}{g}=\frac{g_{\mathrm{rad}}^{\mathrm{CAK}}}{g}$,

and the parameter $\Gamma_{\mathrm{B}}$, which is the ratio of the $\mathrm{CAK}$ radiative force $g_{\mathrm{rad}}^{\mathrm{CAK}}$ to gravity, for the case that dynamical friction reaches a maximum,

$\Gamma_{\mathrm{B}}=\frac{\left.g_{\mathrm{rad}}^{\mathrm{CAK}}\right|_{x_{\mathrm{pi}}=0.968}}{g}$.

Decoupling should occur, if

$\Gamma_{\mathrm{B}}<\Gamma_{\mathrm{L}}$.

From Fig. 2, decoupling is expected in model B5 for $r \gtrsim 1.6 R_{*}$. Analytically, $r_{\mathrm{d}}$ is determined by Springmann \& Pauldrach (1992),

$r_{\mathrm{d}}=R_{*}\left(1-\left(\frac{\dot{M}_{\mathrm{CAK}} \eta k_{\mathrm{pi}} G_{\mathrm{max}}}{4 \pi \beta R_{*} v_{\infty}^{3}}\right)^{1 /(3 \beta-1)}\right)^{-1}$, 

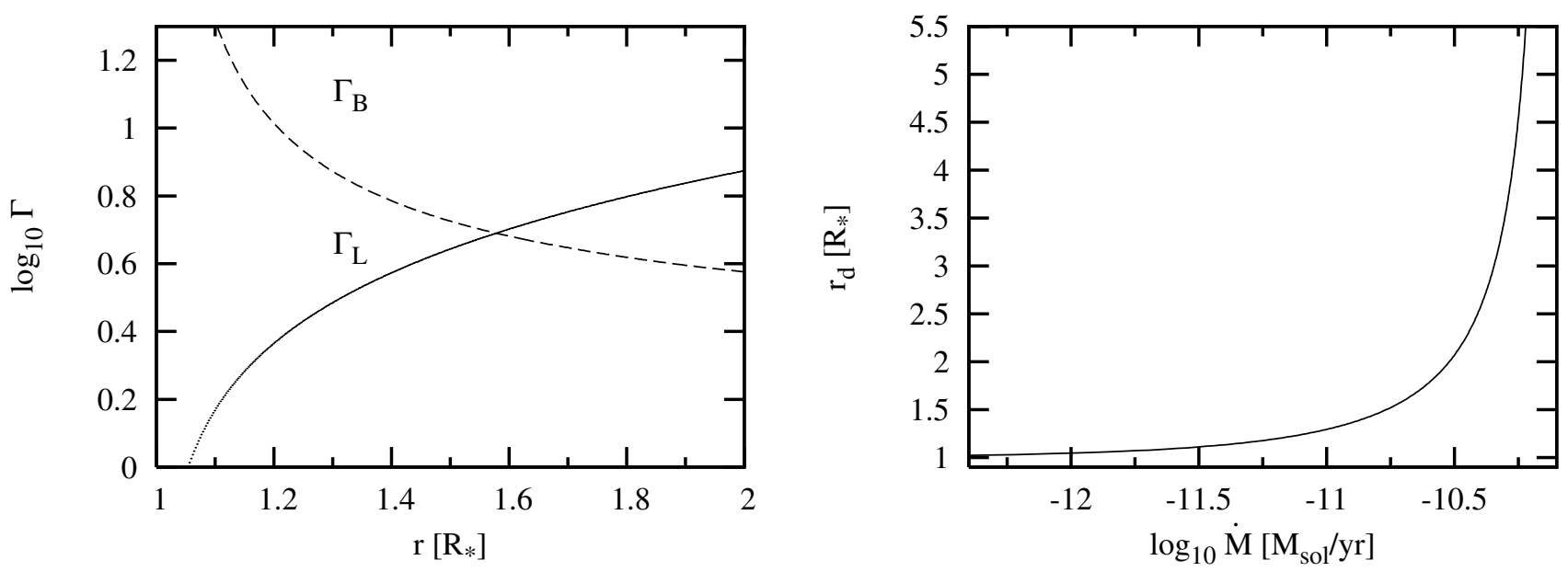

Fig. 2. Left panel: plot of $\Gamma_{\mathrm{B}}$ and $\Gamma_{\mathrm{L}}$ as function of radius for the model B5. The curves cross at $r \sim 1.6 R_{*}$. Right panel: dependence of the decoupling radius $r_{\mathrm{d}}$ on mass-loss rate $\dot{M}$ for the model B5. If the wind is denser, the decoupling radius is located farther away from the star.

Table 1. Parameters of wind models.

\begin{tabular}{cccccccc}
\hline \hline Star & $\begin{array}{c}M \\
{\left[M_{\odot}\right]}\end{array}$ & $\begin{array}{c}T_{\text {eff }} \\
{[\mathrm{K}]}\end{array}$ & $\begin{array}{c}R_{*} \\
{\left[R_{\odot}\right]}\end{array}$ & $\alpha$ & $\mathrm{k}$ & $\delta$ & $q_{\mathrm{i}} / q_{\mathrm{p}}$ \\
& 90.0 & 28500 & 37.0 & 0.590 & 0.170 & 0.09 & 3.0 \\
\hline \hline B0 & 90.01 & & \\
B5 & 4.36 & 15500 & 3.01 & 0.511 & 0.235 & 0.12 & 2.5 \\
\hline
\end{tabular}

Table 2. Calculated values of $v_{\infty, \mathrm{p}}$ and $v_{v_{\infty}, \mathrm{i}}$ in comparison with $v_{\infty}^{\mathrm{CAK}}$ from the one-component model, and derived values of the mass-loss rate for absorbing ions, $\dot{M}_{\mathrm{i}}$, and for the passive plasma, $\dot{M}_{\mathrm{p}}$.

\begin{tabular}{cccccc}
\hline \hline Star & $\begin{array}{c}v_{\infty, \mathrm{p}} \\
{\left[\mathrm{km} \mathrm{s}^{-1}\right]}\end{array}$ & $\begin{array}{c}v_{v_{\infty}, \mathrm{i}} \\
{\left[\mathrm{km} \mathrm{s}^{-1}\right]}\end{array}$ & $\begin{array}{c}v_{\infty}^{\mathrm{CAK}} \\
{\left[\mathrm{km} \mathrm{s}^{-1}\right]}\end{array}$ & $\begin{array}{c}\dot{M}_{\mathrm{i}} \\
{\left[\dot{M}_{\odot} \mathrm{p} . \mathrm{y} .\right]}\end{array}$ & $\begin{array}{c}\dot{M}_{\mathrm{p}} \\
{\left[\dot{M}_{\odot} \mathrm{p} . \mathrm{y} .\right]}\end{array}$ \\
\hline \hline B0 & 1600 & 1600 & 1600 & $6.6 \times 10^{-8}$ & $4.4 \times 10^{-6}$ \\
B5 & 200 & 36000 & 800 & $2.3 \times 10^{-14}$ & $1.5 \times 10^{-12}$ \\
\hline
\end{tabular}

Note 1 . The value of the passive plasma mass-loss rate roughly corresponds to the CAK value.

where $\beta$ is the beta-law parameter (see, e.g., Porter \& Skouza 1999) and $\eta$ has been introduced in (4).

\subsection{Well coupled stellar wind}

For this case we used the B0 model star from Krtička \& Kubát (2000). Our hydrodynamic simulation evolved to a steady mCAK solution with the correct terminal speed $v_{\infty}$. Figure 3 shows the velocity law and the density stratification of the wind. For comparison with Krtička \& Kubát (2000) and the CAK predictions, we summarise the results of our calculation in Table 2. As was expected, for the case of a well-coupled wind, the velocities of both absorbing ions $v_{v_{\infty}, \mathrm{i}}$ and passive plasma $v_{\infty, \mathrm{p}}$ are roughly the same.

The agreement between our results and those obtained with the one-component version of the code is very good, see Fig. 3. There is also good agreement with the stationary calculation by Krtička \& Kubát (2000).

To test the sensitivity of the results to our approximation of the Chandrasekhar function, we used different sets of parameters $a_{2}, b_{2}, c_{2}$ and of points $x_{1}, x_{2}$ without the condition of a best fit. For $a_{2}=-0.176, b_{2}=0.404, c_{2}=-0.001$, $x_{1}=0.15$, and $x_{2}=1.85$, fit $G_{A}$ lies everywhere above $G$, with maximum deviation $\approx 30 \%$, whereas for $a_{2}=-0.224$, $b_{2}=0.438, c_{2}=-0.0033, x_{1}=0.10$, and $x_{2}=1.10$, the function $G_{A}$ lies everywhere below $G$, with maximum deviation $\approx 50 \%$. It turns out that, for the case of a wellcoupled wind, the detailed form of the approximation of the Chandrasekhar function is not very important, since the steadystate wind solutions are always fairly similar. On the other hand, for a poorly coupled wind, a correct approximation is mandatory, since the frictional force determines the point where the wind starts to decouple.

\subsection{Low-density wind with decoupling}

As a second model, we considered the B5 star from Krtička \& Kubát (2000). Their two-component model shows a solution with lower acceleration compared to the normal CAK solution, but did not lead to decoupling. Results of our calculations are shown in Fig. 4. Compared to Krtička \& Kubát (2000), we changed the average ion charge to a slightly higher value $q_{\mathrm{i}}=2.5$ instead of $q_{\mathrm{i}}=2.0$, as before. The aim of this was to increase dynamical friction to prevent the appearance of pulsating shells. Namely, this higher value of $q_{\mathrm{i}}$ shifts the decoupling radius to a location where the escape velocity is lower than the local speed of the passive plasma and, consequently, matter is no longer gravitationally bound to the star. The more subtle case when the matter is still gravitationally bound to the star will be considered in a forthcoming paper.

We find that metal ions decouple from the passive plasma and start to accelerate steeply at the decoupling radius, whereas the passive plasma starts to decelerate at this location. The decoupling point from the simulation agrees roughly with the prediction from expression (32), $r_{\mathrm{d}} \approx 1.6$. (To derive this value, we use the CAK value for $v_{\infty}$ from Table 2 and $\beta=0.8$, which is a good estimate for our model.) The present decoupling contradicts the results of Krtička \& Kubát (2000), who obtained a shallow, coupled solution.

The quite unexpected result of Krtička \& Kubát (2000) was analysed by Owocki \& Puls (2002), who performed a linear stability analysis of the time-dependent hydrodynamic equations to derive perturbation growth rates and propagation speeds in a two-component stellar wind. These authors found that the ion decoupling instability persists for the Krtička \& Kubát (2000) shallow-wind solution, for long wavelength perturbations with 

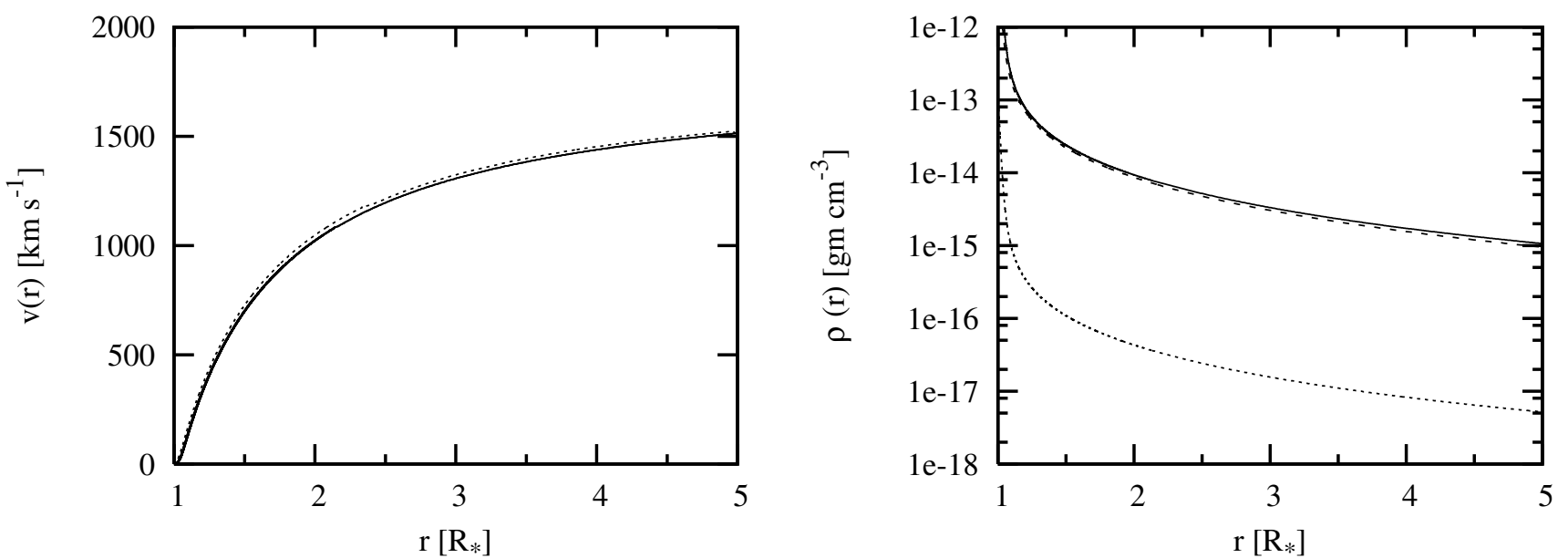

Fig. 3. Left panel: final wind-velocity laws for model $\mathrm{B} 0$ after 55 flow time units $R_{*} / v_{\infty}$. Absorbing ions are marked by (-), passive plasma by $(--)$, and the result of the one-component code by $(\cdots)$. The curves for ions and passive plasma are indistinguishable. Right panel: density for the same model B0.
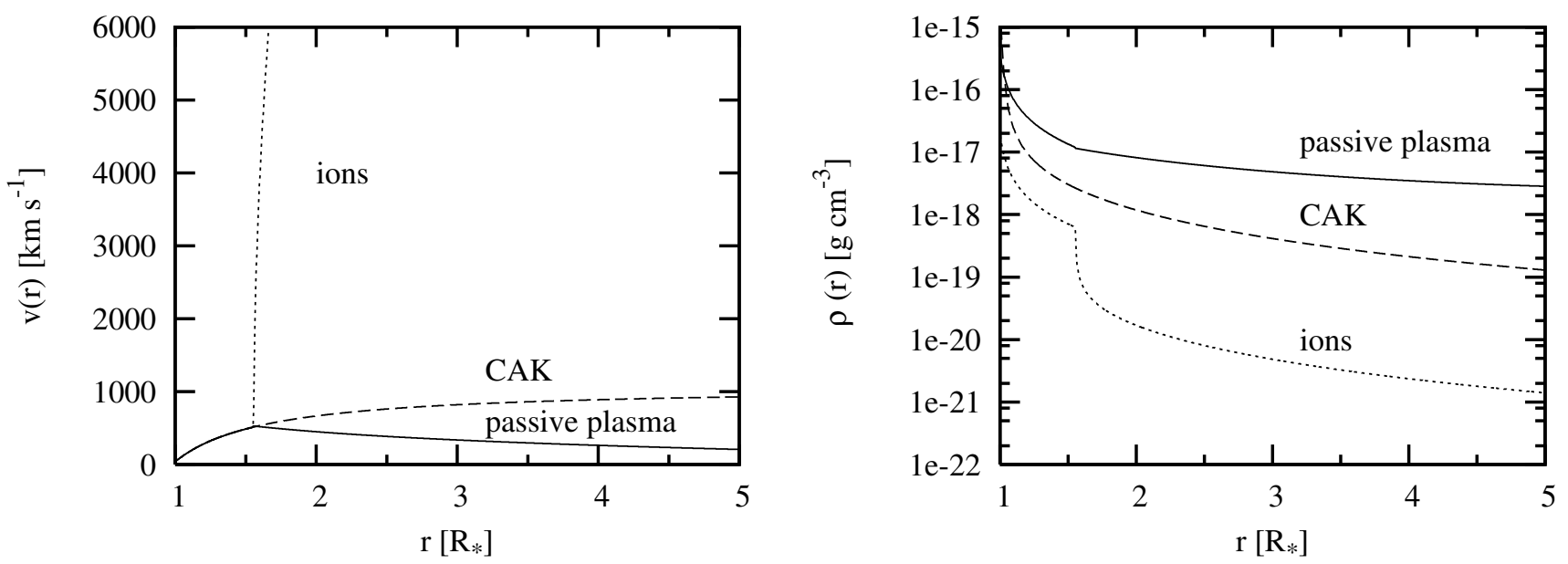

Fig. 4. Left panel: einal wind-velocity laws for model B5 after 55 flow time units $R_{*} / v_{\infty}$. Absorbing ions are marked by $(\cdots)$, passive plasma by (-), and the result of the one-component code by (- -). Right panel: wind density for the same model.

high temporal growth rate $\approx 10^{4} v / R_{*}$. It therefore seems that the Krtička \& Kubát (2000) solution is not a physically valid solution.

Owocki \& Puls (2002) also show that for the solution obtained by Krtička \& Kubát (2000), the ion-decoupling instability has a modest spatial growth rate, mainly due to the fast speed of perturbation propagation, which only allows a slight amplification during the time needed to converge to the steady-state solution. We may speculate that this is related to how Krtička \& Kubát (2000) indeed found convergence of the iteration scheme applied to solve the steady-state equations to a shallow, onecomponent solution.

As a test, we increased the value of the ion charge to a rather unrealistic value of $q_{\mathrm{i}}=5$, which artificially increases the Coulomb coupling. For this case we obtained a stable, onecomponent flow solution according to CAK.

After decoupling, the ion velocity gradient is so large that the associated spectral lines from ions should be optically thin. Because of this dramatic reduction of the Sobolev optical depth in ions, the absorption of this highly accelerated material becomes weak and the signature of this material in the spectrum is also weak. We must mention that the $\mathrm{CAK}$ radiative acceleration given by Eq. (4) is overestimated. Babel (1996) showed that inclusion of the shadowing effect by photospheric lines to radiative acceleration calculation lowers its value significantly for B stars with a low-density wind compared with the CAK model. Thus, the high ion speeds from the above figure would not be observed, and the observed wind speed is instead that of the passive plasma (Springmann \& Pauldrach 1992).

\section{Summary}

We develop a method for time-dependent hydrodynamic simulations of multi-component stellar winds. To avoid resolving the prohibitively short friction time scale (causing very stiff system of equations) in our time-explicit scheme, we used a threeinterval fit to the Chandrasekhar function and solved the friction terms (after operator-splitting) analytically. The stability and accuracy of our method is demonstrated for a B0 model star with a well-coupled wind, where our flow solution evolves to the wellknown steady mCAK solution.

For a B5 model star with a low-density wind, we find that ion decoupling from the passive wind plasma occurs at a definite wind radius, instead of the transition to a shallow, coupled 
wind solution with small acceleration of both components, as predicted by Krtička \& Kubát (2000).

In the future, we will apply the code to a larger sample of stars and study whether pulsating shells may originate in multicomponent stellar winds (Porter \& Skouza 1999).

Acknowledgements. We thank Robert Nikutta and Jiří Krtička for fruitful discussions and comments. This work was supported by grant D/04/25764 from the Deutscher Akademischer Austausch Dienst and grant B301630501 from the Grant Agency of the Academy of Sciences of the Czech Republic, as well as by Deutsche Forschungsgemeinschaft under grant numbers FE 573/2 and FE 573/3. The Astronomical Institute Ondřejov is supported by project AV0 Z10030501.

\section{References}

Abbott, D. C. 1980, ApJ, 242, 1183

Abbott, D. C. 1982, ApJ, 259, 282

Anderson, J. D., Jr. 1995, Computational Fluid Dynamics (New York: McGrawHill Book Co)

Babel, J. 1996, A\&A, 309, 867

Carlberg, R. G. 1980, ApJ, 241, 1131

Castor, J. I. 1974, MNRAS, 169, 279

Castor, J. I., Abbott D. C., \& Klein, R. I. 1975, ApJ, 195, 879

Castor, J. I., Abbott, D. C., \& Klein, R. I. 1976, in Physique des mouvements dans les atmosphères stellaires, ed., R. Cayrel \& M. Sternberg, CNRS Paris, p. 363

Cassinelli, J. P., \& Olson, G. L. 1979, ApJ, 229, 304
Chandrasekhar, S. 1943, Principles of Stellar Dynamics (University of Chicago Press)

Dessart, L., \& Owocki, S. P. 2005, A\&A, 437, 657

Feldmeier, A. 1995, A\&A, 299, 523

Feldmeier A., \& Shlosman I. 2000, ApJ, 532, L125

Friend, D. B., \& Castor J. I. 1983, ApJ, 272, 259

Friend, D. B., \& MacGregor, K. B. 1984, ApJ, 282, 591

Friend, D. B., \& Abbott, D. C. 1986, ApJ, 311, 701

Karlický, M., Brown, J. C., Conway, A. J., \& Penny, G. 2000, A\&A, 353, 729

Krolik, J., \& Raymond, J. C. 1985, ApJ, 298, 660

Krtička, J., \& Kubát, J. 2000, A\&A, 359, 983

Krtička, J., \& Kubát, J. 2001, A\&A, 377, 175

Krtička, J., \& Kubát, J. 2004, A\&A, 417, 1003

LeVeque, R. J., Mihalas, D., Dorfi, E. A., \& Müller, E. 1998, Computational

Methods for Astrophysical Fluid Flow (Berlin: Springer-Verlag)

Lucy, L. B., \& Solomon, P. M. 1970, ApJ, 159, 879

MacGregor, K. B., Hartmann, L., \& Raymond, J. C. 1979, ApJ, 231, 514

Nelson, G. D., \& Hearn, A. G. 1978, A\&A, 65, 223

Owocki, S. P., \& Rybicki, G. B. 1984, ApJ, 284, 337

Owocki, S. P., \& Rybicki, G. B. 1985, ApJ, 299, 265

Owocki, S. P., \& Puls, J. 2002, ApJ, 568, 965

Owocki, S. P., Castor, J. I., \& Rybicki, G. B. 1988, ApJ, 335, 914

Pauldrach, A., \& Puls, J., 1990, RvMA, 3, 124

Pauldrach, A., Puls, J., \& Kudritzki, R. P. 1986, A\&A, 164, 86

Porter, J. M., \& Skouza, B. A. 1999, A\&A, 344, 205

Spitzer, L. 1956, Physics of fully ionized gases (New York: Interscience)

Springmann, U., \& Pauldrach, A. 1992, A\&A, 262, 515

Van Leer, B. 1977, J. Comput. Phys, 89, 439

Prinja R. K., \& Howarth I. D. 1986, ApJS, 61, 357 